\title{
Transient auroral features at Saturn: Signatures of energetic particle injections in the magnetosphere
}

\author{
A. Radioti, ${ }^{1}$ D. Grodent, ${ }^{1}$ J.-C. Gérard, ${ }^{1}$ E. Roussos,${ }^{2}$ C. Paranicas, ${ }^{3}$ B. Bonfond, ${ }^{1}$ \\ D. G. Mitchell, ${ }^{3}$ N. Krupp, ${ }^{2}$ S. Krimigis, ${ }^{3,4}$ and J. T. Clarke ${ }^{5}$
}

Received 22 July 2008; revised 16 December 2008; accepted 12 January 2009; published 21 March 2009.

[1] We report for the first time transient isolated auroral spots at Saturn's southern polar region, based on Hubble Space Telescope (HST) FUV images. The spots last several minutes and appear distinct from the rest of the auroral emissions. We study two sets of HST and Cassini observations during which Cassini instrumentation detected signatures of energetic particle injections close to the region where, on the same day, HST observed transient auroral spots. On the basis of the simultaneous remote and in situ observations, we discuss the possibility that the transient features are associated with the dynamical processes taking place in the Kronian magnetosphere. Given the limitations in the available observations, we suggest the following possible explanations for the transient aurora. The injection region could directly be coupled to Saturn's ionosphere by pitch angle diffusion and electron scattering by whistler waves, or by the electric current flowing along the boundary of the injected cloud. The energy contained in the injection region indicates that electron scattering could account for the transient aurora process.

Citation: Radioti, A., D. Grodent, J.-C. Gérard, E. Roussos, C. Paranicas, B. Bonfond, D. G. Mitchell, N. Krupp, S. Krimigis, and J. T. Clarke (2009), Transient auroral features at Saturn: Signatures of energetic particle injections in the magnetosphere, J. Geophys. Res., 114, A03210, doi:10.1029/2008JA013632.

\section{Introduction}

[2] Saturn's ultraviolet auroral emissions were shown to differ morphologically from those of Earth and Jupiter [Clarke et al., 2005]. Some auroral features appear to be in partial corotation, whereas others are fixed with respect to the solar wind direction. Studies of southern FUV aurorae [Gérard et al., 2004] based on Hubble Space Telescope (HST) images during 1997-2001 showed that the morphology and brightness of the Kronian aurora varies on time scales of hours, and it often forms a spiral shape around the pole. Bright aurorae were observed to expand significantly poleward in the dawn sector such that the area of the polar cap was much reduced [Clarke et al., 2005]. An extensive study based on 64 FUV HST images demonstrated that the bright ring of auroral emission consists of several arcs of different width and brightness and forming along different parallels [Grodent et al., 2005]. Theoretical studies suggested that the morphology of Saturn's aurora is, to a large

\footnotetext{
${ }^{1}$ Laboratoire de Physique Atmosphérique et Planétaire, Institut d'Astrophysique et de Géophysique, Université de Liège, Liege, Belgium.

${ }^{2}$ Max-Planck-Institut für Sonnensystemforschung, Katlenburg-Lindau, Germany.

${ }^{3}$ Applied Physics Laboratory, Johns Hopkins University, Laurel, Maryland, USA.

${ }^{4}$ Office for Space Research and Applications, Academy of Athens, Athens, Greece.

${ }^{5}$ Center for Space Physics, Boston University, Boston, Massachusetts, USA.

Copyright 2009 by the American Geophysical Union. 0148-0227/09/2008JA013632\$09.00
}

extent, controlled by the balance between the magnetic field reconnection rate at the dayside magnetopause and the reconnection rate in the nightside tail [Cowley et al., 2004]. Additionally, the combined action of reconnection and rotation naturally gives rise to spiral structures on newly opened and newly closed field lines [Cowley et al., 2005]. Recent analysis indicated that the quasi-continuous main oval is produced by the magnetosphere-solar wind interaction, through the shear in rotational flow across the open closed field line boundary [Bunce et al., 2008b]. Saur et al. [2006] reported electron beam observations in the magnetosphere of Saturn and compared them statistically with auroral images. The energy contained in the beams suggested that upward acceleration is an essential part of the overall auroral process.

[3] In the present study we focus on transient auroral features and discuss them in terms of the dynamics taking place in the Kronian magnetosphere. Spatial and temporal variability of energetic particle flux is important in the magnetospheric-ionospheric processes at the Jovian system. Mauk et al. [2002] suggested that energetic particle injections observed in Jupiter's magnetosphere trigger transient auroral emissions. Furthermore, short-lived auroral features because of their observed location and their periodic cycle are associated with internally driven reconnection processes in the Jovian magnetotail [Grodent et al., 2004; Radioti et al., 2008].

[4] Cassini data indicated that Saturn's inner magnetosphere is clearly rotation-dominated and provided evidence of widespread occurrence of energetic particle injections [Mauk et al., 2005; Hill et al., 2005; Burch et al., 2005], 


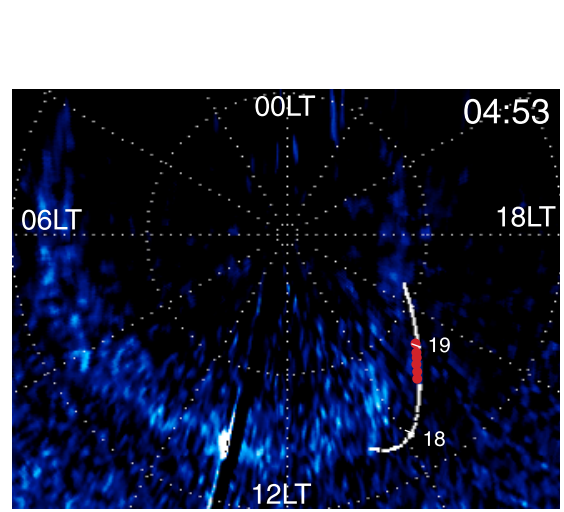

\section{January 18,2007}
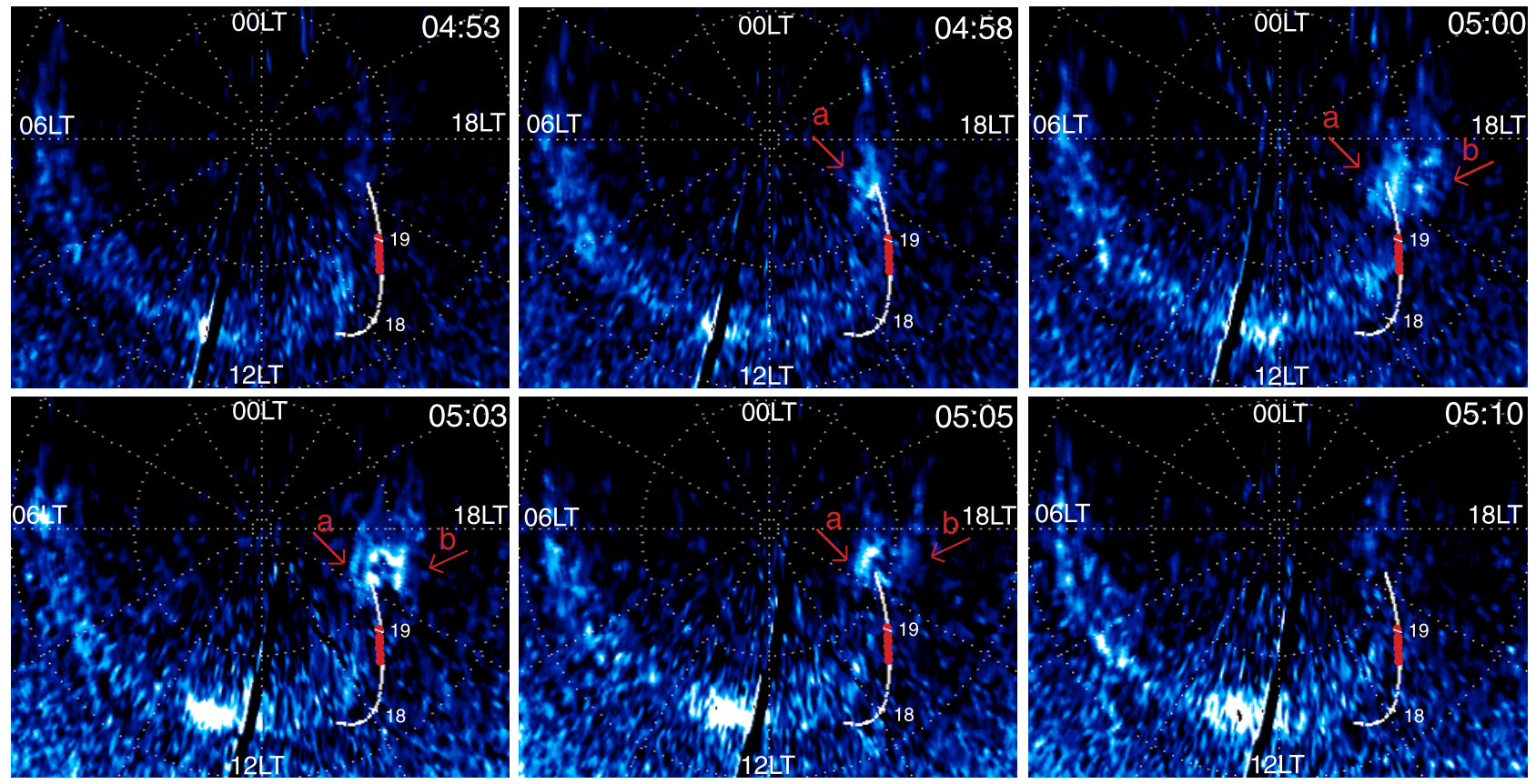

Figure 1. Sequence of six HST/ACS UV images of Saturn's southern aurora obtained on 18 January 2007. The images are projected onto a local time polar grid as viewed through the planet looking from the north, with noon at the bottom and dusk to the right. Isolated transient spots are indicated by "a" and "b." All images share the same brightness scale. The brightness is not corrected for the limb-brightening effect. The ionospheric footprint of Cassini trajectory during the 17 to 19 January 2007 time interval is shown by the solid white line. The mapped spacecraft position at the time during which Cassini observed signatures of energetic particle injections is indicated by red. For the magnetic mapping we use a combination of the "Cassini" internal field model for Saturn [Dougherty et al., 2005] and a typical ring current model [Bunce et al., 2007, 2008a].

similar to those occurring at Jupiter. The injection signatures include drift dispersion events, in which electrons and ions of various energies are separated azimuthally with time. They are possibly powered by centrifugal interchange events in which distant magnetic flux tubes containing hot, tenuous plasma convect inward to replace flux tubes containing dense, cold plasma. Evidence of plasma heating in the magnetosphere of Saturn is given by ENA (Energetic Neutral Atoms) emissions, possibly associated with magnetospheric substorms [Mitchell et al., 2005]. Further studies confirmed the presence of rapid tail reconnection and implied that plasma is accelerated toward and away from the planet [Jackman et al., 2007]. Statistical analyses based on 48 injection events detected by Cassini showed that they occur randomly at all local times and their age ranges from $\sim 1 / 2$ hours to tens of hours [Hill et al., 2005]. Energetic particle injections play an important role in the population of Saturn's magnetosphere. It is observed that the electron population $(20-410 \mathrm{keV})$ in the inner magnetosphere results principally from recent injections [Paranicas et al., 2007]. Plasma wave measurements within local plasma injections in the magnetosphere of Saturn showed the presence of whistler waves and the existence of a narrow loss cone in the injection region [Menietti et al., 2008]. The authors mentioned the possibility that the injection regions could be linked to the Kronian ionosphere.

\section{Simultaneous HST and Cassini Observations}

[5] During January 2007 and February 2008, daily UV observations of Saturn's southern aurora were obtained with the Advanced Camera for Surveys (ACS) on board HST, as part of a coordinated HST-Cassini campaign. Each set of observations (HST orbit) lasts $\sim 45 \mathrm{~min}$ during which 19 consecutive images are taken every $2-3 \mathrm{~min}$. This data set makes it possible for the first time to study short-lived features of the order of a few minutes in the saturnian aurora. The angular resolution of the images is 0.08 arcsec FWHM point spread function. The determination of the planetary center on the images required for the polar projections was provided by an automatic ring fitting procedure. In this work we study two cases of simultaneous HST and Cassini observations. We use ion and electron measurements from two of the three Magnetospheric Imaging Instrument (MIMI) on board the Cassini spacecraft, the Energy Mass Spectrometer (CHEMS) sensor and the Low Energy Magnetospheric Measurements System (LEMMS) sensor. Details of the MIMI instrumentation are given by Krimigis et al. [2004]. 


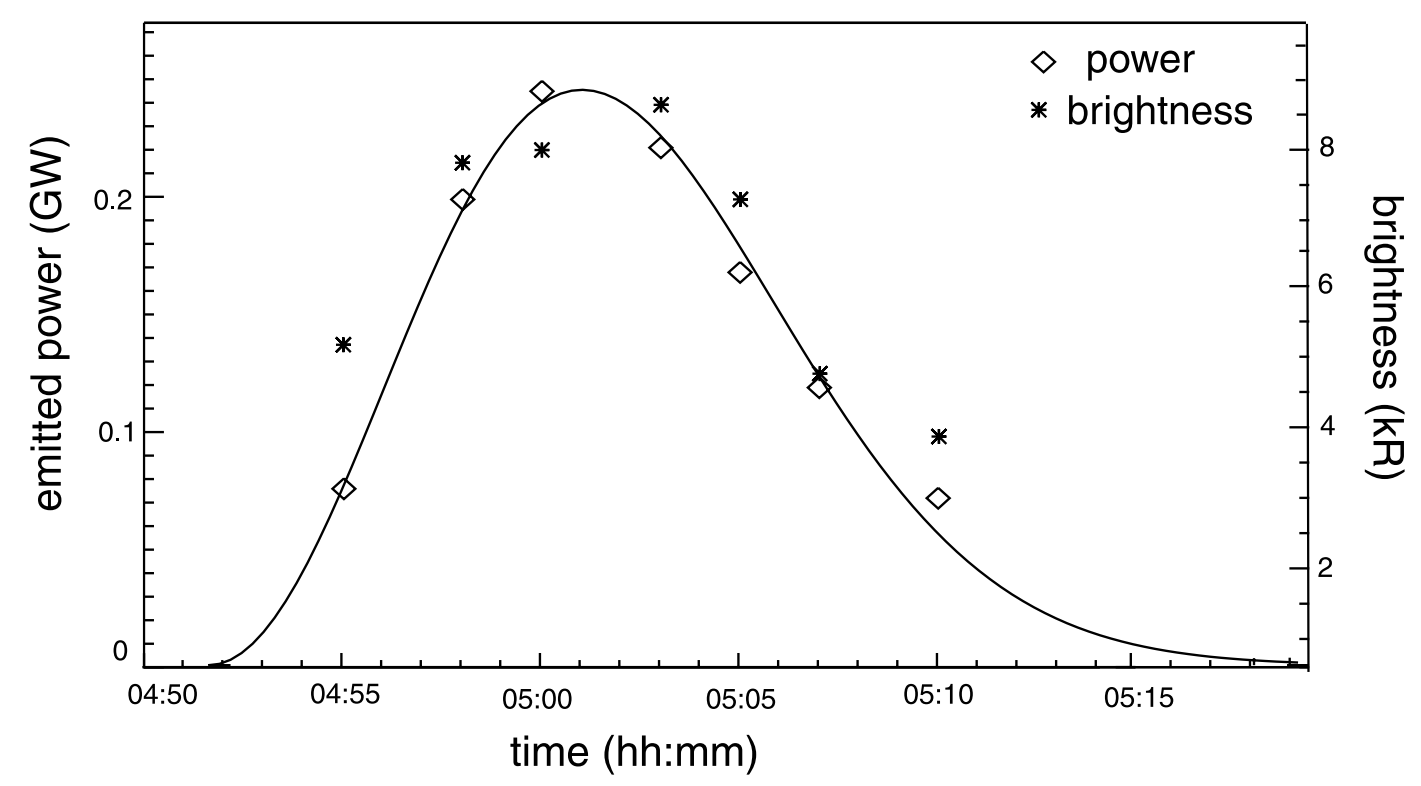

Figure 2. Time evolution of the emitted power and the maximum brightness of spot "a" (Figure 1) indicated by diamonds and stars, respectively. The solid line shows the Maxwellian fit of the emitted power. The brightness is above the background emission and corrected for the limb-brightening effect using $\cos \phi$ factor, where $\phi$ is the zenithal angle.

\subsection{Observations on 18 January 2007}

[6] Figure 1 shows a sequence of six HST/ACS UV images of Saturn's southern aurora obtained on 18 January 2007. The images are projected onto a polar local time grid as viewed through the planet looking from the north, with noon at the bottom and dusk to the right. They are taken every 2-3 $\mathrm{min}$ and show the evolution of the isolated auroral spots, marked with "a" and "b." Spots "a" and "b" last for 15 and $7 \mathrm{~min}$, respectively, and they move in the corotation direction. The measured maximum brightness of the auroral spots above the background emission and corrected for the limb-brightening effect (using $\cos \phi$ factor, where $\phi$ is the zenithal angle) is $\sim 9 \mathrm{kR}$. The time evolution of the emitted power and the maximum brightness of spot "a" is shown in Figure 2 with the diamonds and stars symbols, respectively. The solid line indicates the Maxwellian fit of the emitted power. On top of the images in Figure 1 the solid line shows the ionospheric footprint of Cassini trajectory, during the time interval 17 to 19 January 2007. Cassini was in the postnoon local time sector moving from subspacecraft latitude of $-10^{\circ}$ to $45^{\circ}$. For the magnetic mapping we use a combination of the "Cassini" internal field model for Saturn [Dougherty et al., 2005] and a typical ring current model [Bunce et al., 2007, 2008a]. The ring current model is employed for subsolar magnetopause position of $\sim 21 R_{s}$.

[7] On the same day that HST observed the auroral spots, a few hours later (from 1330 UT, day 18 to 0130 UT, day 19), Cassini observed signatures of energetic particle injections. Figure 3 shows ion data from the MIMI CHEMS sensor on board the Cassini spacecraft. The energy-time spectrogram of Figure 3 shows that higher-energy ions arrive at the spacecraft before the low-energy ions. Those features are identified as energetic particle injections. The LEMMS electron sensor on board Cassini did not observe simultaneous injection signatures in the electron data set. The visibility of the feature depends on the magnitude of the radial gradient in the phase space density which varies according to species, as a result, injection signatures seen at the ion data set are not always simultaneously detected in the electron data set. The mapped spacecraft position at the time during which Cassini observed signatures of energetic particle injections is shown in red in Figure 1, close to the observed isolated UV auroral spots.

\subsection{Observations on 7 and 8 February 2008}

[8] On 7 and 8 February 2008, HST observed transient auroral features similar to those reported on 18 January 2007. Figure 4 shows Saturn's southern aurora on 7 and 8 February 2008 with the same format as Figure 1. In each image, the transient auroral spots are indicated with "a," "b" and "c" and last for 30,12 and 26 min, respectively. Their measured maximum brightness above the background emission and corrected for the limb-brightening effect is $\sim 10 \mathrm{kR}$. The ionospheric footprint of Cassini trajectory during the time interval of 7 and 8 February 2008 is depicted with the solid line. Cassini was moving from postnoon to dusk sector at subspacecraft latitude range of $39^{\circ}$ to $47^{\circ}$

[9] On 8 February 2008 (from 1200 UT to 1600 UT), Cassini LEMMS instrumentation measured energy dispersed features identified as signatures of energetic particle injections (Figure 5). The energy-time spectrogram shows the presence of fainter traces at higher energies (in yellow, green and blue). These traces have an arc-like shape that disappears at small radial distances, indicative of injections that have aged for several days [Hill et al., 2005; Paranicas et al., 2007]. The mapped spacecraft position at the time during which Cassini observed signatures of energetic particle injections, is shown in red in Figure 4. A possible auroral counterpart at the exact location of the observed 


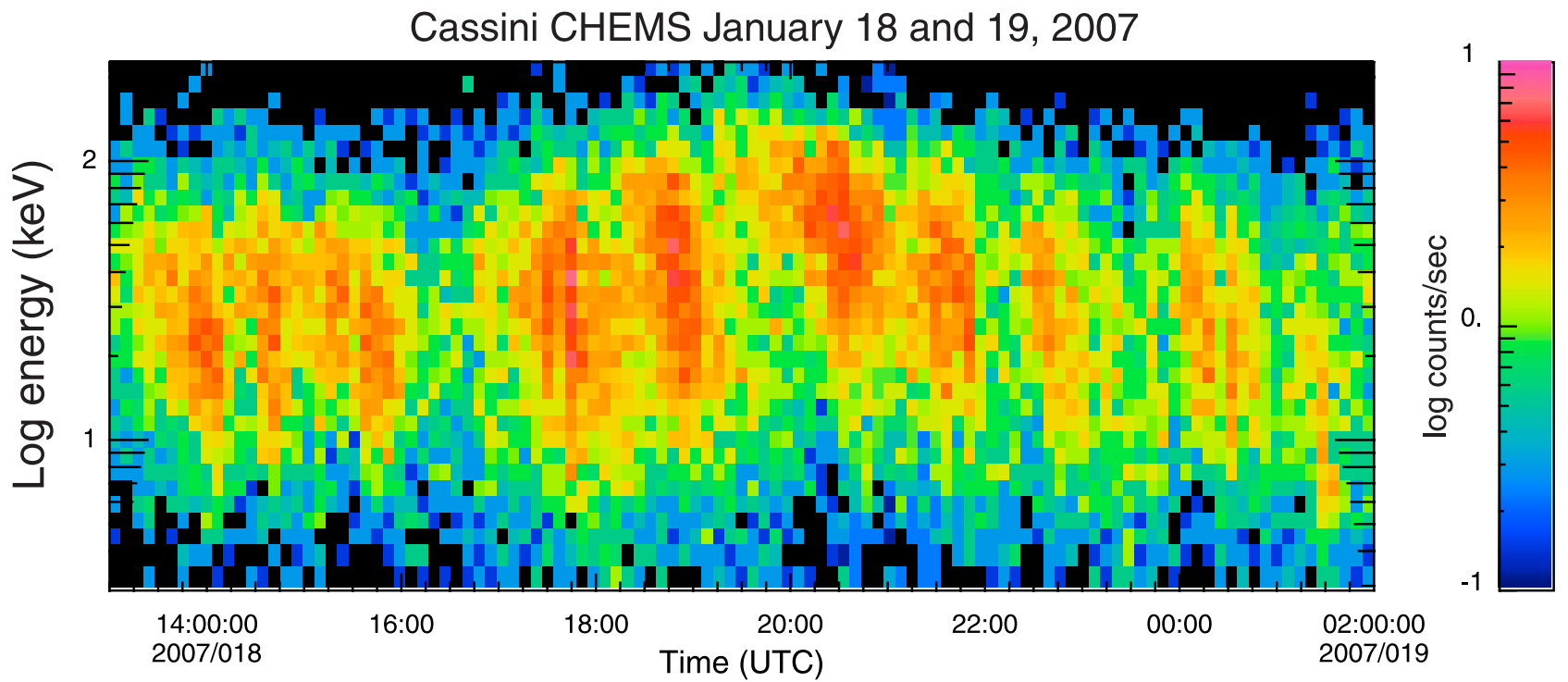

Figure 3. Energy-time spectrogram from the Cassini CHEMS sensor during 18 and 19 January 2007, showing ion counts/s detected over a wide range of energies, indicative of injection signatures.

injections, could not be observed because of HST improper viewing geometry.

[10] We model the individual faint traces of the injections and derive their initiation time and location, on the basis of an analysis similar to that described by Paranicas et al. [2007]. The model assumes that electrons are simultaneously injected at all radial distances and do not drift radially. This period was not an ideal one to study from the point of view of LEMMS because of changes in pitch angle sampling. We were able to perform an approximate, nonunique fit corresponding to an injection on 6 February 2008 at $0600 \mathrm{UT}$, around noon local time. The injected population travels around the entire planet in more than $10 \mathrm{~h} 46 \mathrm{~min}$ and disperses. At the radial distances of interest to us here (which correspond to the latitudes of the observed auroral spots), the plasma corotation speed is well below rigid and the field is nondipolar. Therefore, we are not in a position to predict how exactly the injected population evolves. The auroral spots observed on 7 and 8 February (Figure 4) could possibly be associated with the injected population initiated on 6 February and its faint traces were still observed by Cassini on 8 February.

\section{Discussion}

[11] In this work we present cases of isolated transient spots, for the first time reported in the aurora of Saturn. Similar auroral spots with those presented here, are observed in 13\% of the HST orbits in the 2007-2008 data set (each orbit lasts $\sim 45 \mathrm{~min}$ ) and are only detected where there
February 7, 2008

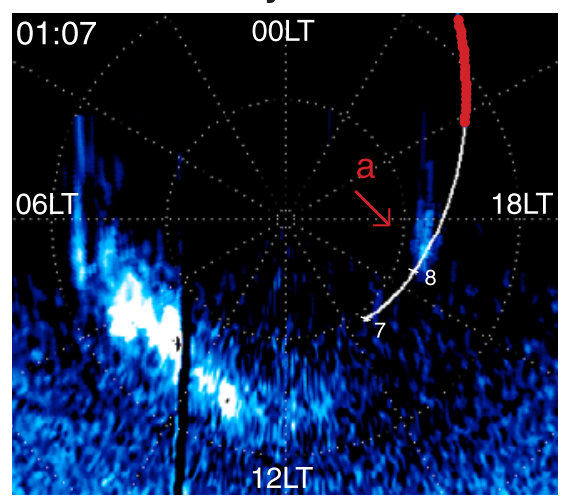

February 7, 2008

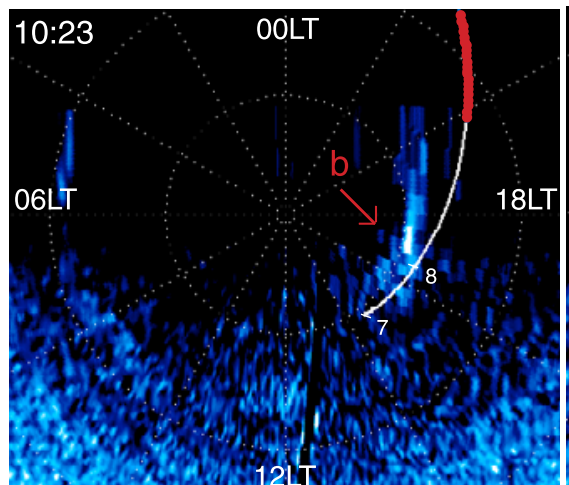

February 8, 2008

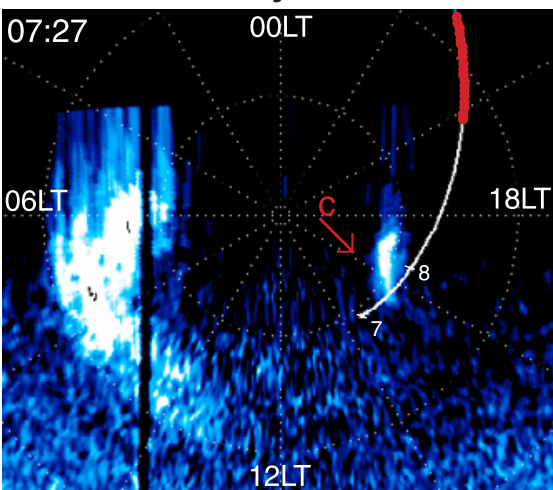

Figure 4. Three HST/ACS UV images of Saturn's southern aurora obtained on 7 and 8 February 2008. The images have the same format as in Figure 1. Isolated transient spots are indicated in each image with "a," "b," and "c" arrows. The ionospheric footprint of Cassini trajectory during the 7 and 8 February 2008 time interval is shown by the solid line. The mapped spacecraft position at the time during which

Cassini observed signatures of energetic particle injections is indicated by red. 


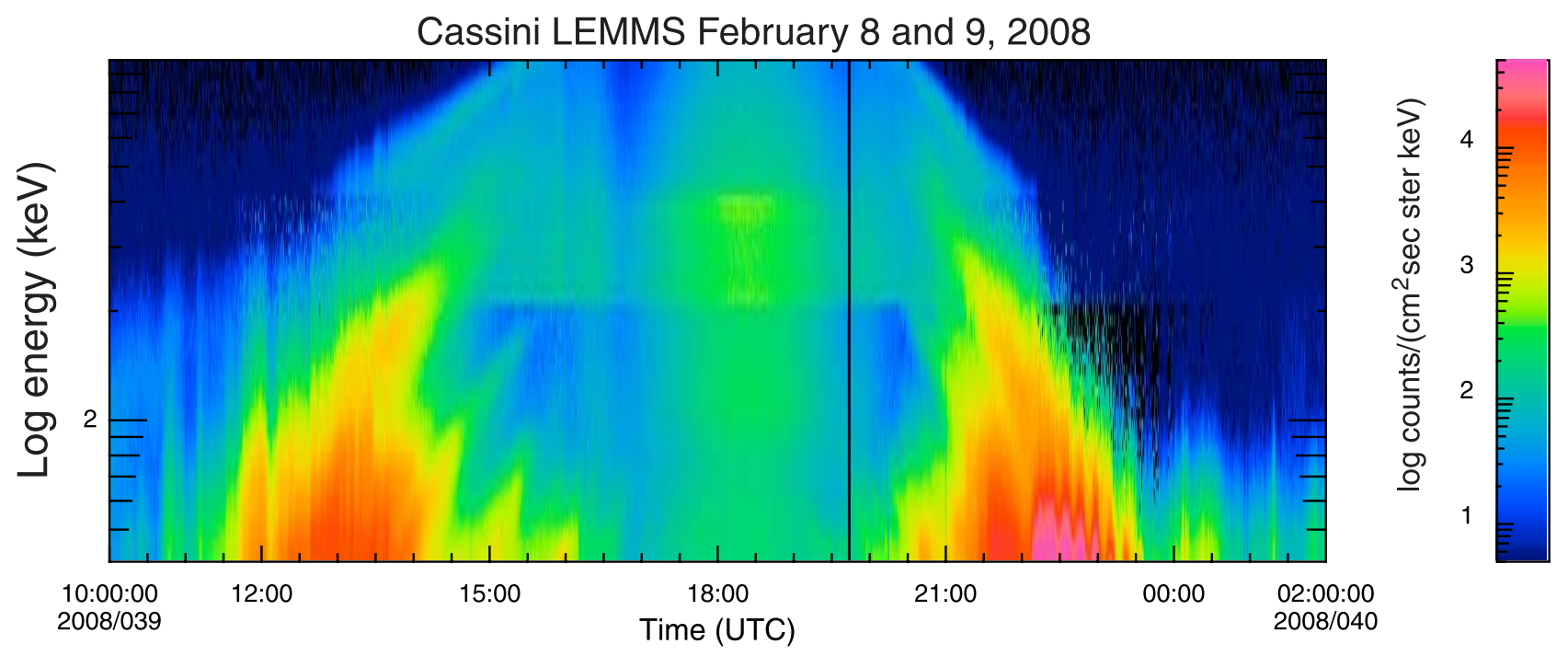

Figure 5. Energy-time spectrogram from the Cassini LEMMS sensor during 8 and 9 February 2008, showing electron fluxes detected over a wide range of energies. The arc-like traces are indicative of injections that aged for a couple of days, which correspond to dates of the HST images shown in Figure 4.

is no other intense auroral emission. Unfortunately, during some time intervals, we do not have simultaneous Cassini observations in the appropriate magnetospheric region, accordingly these cases have not been considered in the present study. On the basis of the simultaneous remote and in situ observations under study, we discuss the possibility that the transient aurora is associated with the energetic particle injections in the magnetosphere of Saturn.

[12] As it is shown at Jupiter, the energetic particle injections can cause transient auroral emissions [Mauk et al., 2002]. The rapid influx of anisotropic energetic electrons provides a source of free energy for the generation of whistler waves [Bolton et al., 1997]. Whistler waves interact with electrons and can lead to pitch angle diffusion and electron precipitation into the ionosphere. In a recent study, Menietti et al. [2008] reported the presence of whistler waves within local plasma injections in the magnetosphere of Saturn. Additionally, the plasma wave measurements strongly suggested the existence of a narrow electron loss cone in the injection region [Menietti et al., 2008].

[13] In order to further investigate the injection region as a source of auroral emission we calculate the precipitation energy flux that can be provided to the ionosphere assuming pitch angle diffusion and scattering of electrons by whistler waves. We calculate the precipitation energy flux [Thorne, 1983] supplied by the energetic electrons to the ionosphere, using an electron spectra sample during the injection event on 8 February 2008. Figure 6 shows the energy spectra measured by LEMMS in the energy range 25 to $609 \mathrm{keV}$ at the onset and during the injection event, indicative of a lowand high-energy spectra along the injection region. It is assumed that the energetic electron intensity measured outside the loss cone by LEMMS is comparable to that within the loss cone itself. We assume that $\sim 1 \%$ of the electrons are scattered, consistent with a narrow loss cone of $\sim 3^{\circ}$ for the injection region [Menietti et al., 2008] and we estimate the precipitation energy flux in the ionosphere (at low altitudes) to range between $\sim 0.5$ and $6.4 \mathrm{~mW} \mathrm{~m}^{-2}$. This energy precipitation flux supports an auroral brightness of 5 to $64 \mathrm{kR}$, considering that $1 \mathrm{~mW} \mathrm{~m}^{-2}$ of injected electrons corresponds to $10 \mathrm{kR}$ [Grodent et al., 2001]. The energy flux derived on the basis of Cassini measurements serves as an indication of the energy that can be precipitated in the ionosphere along an injection region. A comparison of the derived energy precipitation flux $(5-64 \mathrm{kR})$ and the observed brightness of the spots under study $(\sim 9-10 \mathrm{kR})$ shows that the energy contained in the injection region is large enough to account for the transient auroral emissions.

[14] Apart from the scattering process, injected particles can also generate auroral emissions through the electric

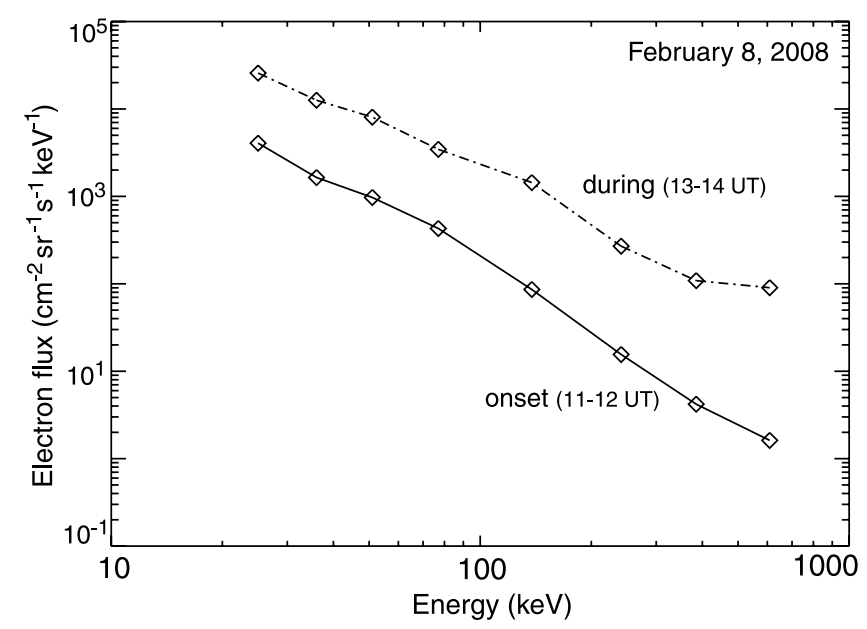

Figure 6. Electron energy spectra at the onset (solid line) and during (dashed line) the injection event for the energy range 25 to $609 \mathrm{keV}$ measured by LEMMS on 8 February 2008. The chosen spectra are indicative of low- and highenergy spectra along the injection region. 
current flowing along the boundary of the injected cloud of hot plasma, as has been suggested for the magnetosphere of the Earth [Kivelson and Russel, 1995]. Currents are driven along the magnetic field lines toward and away from Saturn and can interact strongly with the plasma close to the planet, leading to downward accelerated electrons and auroral emission.

[15] Similarly to Jupiter, we discuss the possibility the transient aurora at Saturn to be associated with tail reconnection. At Jupiter, the observed location of the auroral spots in the nightside and dawn sector as well as their periodic reoccurrence strongly suggest an association of the transient aurora with the reconnection processes taking place in the Jovian magnetotail [Grodent et al., 2004; Radioti et al., 2008]. At Saturn, there is not clear evidence so far that the transient auroral features can be directly linked to tail reconnection. However, plasma heating processes such as rapid bursts of tail reconnection could power the injection events. Evidence of plasma heating has been given by ENA observations, possibly associated with magnetospheric substorms [Mitchell et al., 2005]. Furthermore, magnetic field measurements showed the presence of rapid tail reconnection in the Kronian magnetotail and suggested that the reconnection process accelerates ions and electrons both toward and away from the planet [Jackman et al., 2007]. The heated population is then picked up in the corotation flow around the planet. Since the reconnection processes could power the injections, it is possible that the transient aurora at Saturn bears a signature of tail reconnection.

\section{Conclusions}

[16] On the basis of the HST 2007-2008 observation campaign we report, for the first time, the presence of isolated transient UV auroral spots in the Kronian ionosphere. They last 7 to $30 \mathrm{~min}$, have a maximum brightness of $\sim 10 \mathrm{kR}$ and an emitted power of 0.1 to $0.2 \mathrm{GW}$. Two sets of HST and Cassini observations are examined, during which Cassini instrument detected signatures of energetic particle injections close to the region where, on the same day, HST observed transient auroral spots. Similar transient auroral features observed at Jupiter are shown to be caused by energetic particle injections [Mauk et al., 2002] and other at higher latitudes are suggested to be related to the reconnection processes in the Jovian magnetotail [Grodent et al., 2004; Radioti et al., 2008]. Likewise, we discuss the possibility the auroral spots at Saturn to be associated with the dynamics taking place in the Kronian magnetosphere. Given the limitations in the available observations we suggest the following possible mechanisms for the transient aurora. The injection region can directly be coupled to the Kronian ionosphere by pitch angle diffusion and electron scattering by whistler waves, or by the electric current flowing along the boundary of the injected hot cloud. The energy contained in the injection region shows that electron scattering can account for the transient aurora process.

[17] Acknowledgments. This work is based on observations with the NASA/ESA Hubble Space Telescope, obtained at the Space Telescope Science Institute (STScI), which is operated by AURA, Inc., for NASA under contract NAS5-26555. A.R., D.G., J.-C.G., and B.B. are supported by the Belgian Fund for Scientific Research (FNRS) and the PRODEX
Programme managed by the European Space Agency in collaboration with the Belgian Federal Science Policy Office. J.T.C. is supported by NASA through grant HST-60-10802,01-A to Boston University. The authors would like to thank Emma Bunce for providing the magnetically mapped footprints of Cassini trajectory on the ionosphere and Adem Saglam for the automatic ring fitting procedure required for the polar projections.

[18] Zuyin $\mathrm{Pu}$ thanks W. R. Pryor and another reviewer for their assistance in evaluating this paper.

\section{References}

Bolton, S. J., R. M. Thorne, D. A. Gurnett, W. S. Kurth, and D. J. Williams (1997), Enhanced whistler-mode emissions: Signatures of interchange motion in the Io torus, Geophys. Res. Lett., 2(17), 2123-2126.

Bunce, E. J., S. W. H. Cowley, I. I. Alexeev, C. S. Arridge, M. K. Dougherty, J. D. Nichols, and C. T. Russel (2007), Cassini observations of the variations of Saturn's ring current parameters with system size, J. Geophys. Res., 112, A10202, doi:10.1029/2007JA012275.

Bunce, E. J., C. S. Arridge, S. W. H. Cowley, and M. K. Dougherty (2008a), Magnetic field structure of Saturn's dayside magnetosphere and its mapping to the ionosphere: Results from ring current modeling, J. Geophys. Res., 113, A02207, doi:10.1029/2007JA012538.

Bunce, E. J., et al. (2008b), Origin of Saturn's aurora: Simultaneous observations by Cassini and the Hubble Space Telescope, J. Geophys. Res., 113, A09209, doi:10.1029/2008JA013257.

Burch, J. L., J. Goldstein, T. W. Hill, D. T. Young, F. J. Crary, A. J. Coates, N. André, W. S. Kurth, and E. C. Sittler Jr. (2005), Properties of local plasma injections in Saturn's magnetosphere, Geophys. Res. Lett., 32, L14S02, doi:10.1029/2005GL022611.

Clarke, J. T., et al. (2005), Morphological differences between Saturn's ultraviolet aurorae and those of Earth and Jupiter, Nature, 433, 717-719.

Cowley, S. W. H., E. J. Bunce, and J. M. O'Rourke (2004), A simple quantitative model of plasma flows and currents in Saturn's polar ionosphere, J. Geophys. Res., 109, A05212, doi:10.1029/2003JA010375.

Cowley, S. W. H., S. V. Badman, E. J. Bunce, J. T. Clarke, J.-C. Gérard, D. Grodent, C. M. Jackman, S. E. Milan, and T. K. Yeoman (2005), Reconnection in a rotation-dominated magnetosphere and its relation to Saturn's auroral dynamics, J. Geophys. Res., 110, A02201, doi:10.1029/ 2004JA010796.

Dougherty, M. K., et al. (2005), Cassini magnetometer observations during Saturn orbit insertion, Science, 307, 1266-1270.

Gérard, J.-C., D. Grodent, J. Gustin, A. Saglam, J. T. Clarke, and J. T. Trauger (2004), Characteristics of Saturn's FUV aurora observed with the Space Telescope Imaging Spectrograph, J. Geophys. Res., 109, A09207, doi:10.1029/2004JA010513.

Grodent, D., J. H. Waite Jr., and J.-C. Gérard (2001), A self-consistent model of the Jovian auroral thermal structure, J. Geophys. Res., 106 $12,933-12,952$

Grodent, D., J.-C. Gérard, J. T. Clarke, G. R. Gladstone, and J. H. Waite Jr. (2004), A possible auroral signature of magnetotail reconnection process on Jupiter, J. Geophys. Res., 109, A05201, doi:10.1029/2003JA010341.

Grodent, D., J.-C. Gérard, S. W. H. Cowley, E. J. Bunce, and J. T. Clarke (2005), Variable morphology of Saturn's southern ultraviolet aurora, J. Geophys. Res., 110, A07215, doi:10.1029/2004JA010983.

Hill, T. W., et al. (2005), Evidence for rotationally driven plasma transport in Saturn's magnetosphere, Geophys. Res. Lett., 32, L14S10, doi:10.1029/2005GL022620.

Jackman, C. M., C. T. Russell, D. J. Southwood, C. S. Arridge, N. Achilleos, and M. K. Dougherty (2007), Strong rapid dipolarizations in Saturn's magnetotail: In situ evidence of reconnection, Geophys. Res. Lett., 34, L11203, doi:10.1029/2007GL029764.

Krimigis, S. M., et al. (2004), Magnetosphere imaging instrument (MIMI) on the Cassini mission to Saturn/Titan, Space Sci. Rev., 114, 233-329.

Kivelson, M. G., and C. T. Russel (1995), Introduction to Space Physics, Cambridge Univ. Press, Cambridge, U. K.

Mauk, B. H., J. T. Clarke, D. Grodent, J. H. Waite Jr., C. P. Paranicas, and D. J. Williams (2002), Transient aurora on Jupiter from injections of magnetospheric electrons, Nature, 415, 1003-1005.

Mauk, B. H., et al. (2005), Energetic particle injections in Saturn's magnetosphere, Geophys. Res. Lett., 32, L14S05, doi:10.1029/2005GL022485.

Menietti, J. D., O. Santolik, A. M. Rymer, G. B. Hospodarsky, A. M. Persoon, D. A. Gurnett, A. J. Coates, and D. T. Young (2008), Analysis of plasma waves observed within local plasma injections seen in Saturn's magnetosphere, J. Geophys. Res., 113, A05213, doi:10.1029/ 2007JA012856.

Mitchell, D. G., et al. (2005), Energetic ion acceleration in Saturn's magnetotail: Substorms at Saturn?, Geophys. Res. Lett., 32, L20S01, doi:10.1029/2005GL022647.

Paranicas, C., D. G. Mitchell, E. C. Roelof, B. H. Mauk, S. M. Krimigis, P. C. Brandt, M. Kusterer, F. S. Turner, J. Vandegriff, and N. Krupp 
(2007), Energetic electrons injected into Saturn's neutral gas cloud, Geophys. Res. Lett., 34, L02109, doi:10.1029/2006GL028676.

Radioti, A., D. Grodent, J.-C. Gérard, B. Bonfond, and J. T. Clarke (2008), Auroral polar dawn spots: Signatures of internally driven reconnection processes at Jupiter's magnetotail, Geophys. Res. Lett., 35, L03104, doi: 10.1029/2007GL032460.

Saur, J., et al. (2006), Anti-planetward auroral electron beams at Saturn, Nature, 439, 699-702, doi:10.1038/nature04401.

Thorne, R. M. (1983), Microscopic plasma processes in the Jovian magnetosphere, in Physics of the Jovian Magnetosphere, edited by A. J. Dessler, pp. 454-488, Cambridge Univ. Press, New York.

B. Bonfond, J.-C. Gérard, D. Grodent, and A. Radioti, Laboratoire de Physique Atmosphérique et Planétaire, Institut d'Astrophysique et de
Géophysique, Université de Liège, Allée du 6 Août, 17, Sart-Tilman, B-4000 Liège, Belgium. (a.radioti@ulg.ac.be)

J. T. Clarke, Center for Space Physics, Boston University, 25 Commonwealth Avenue, Room 506, Boston, MA 02215, USA.

S. Krimigis, D. G. Mitchell, and C. Paranicas, Applied Physics Laboratory, Johns Hopkins University, 11100 Johns Hopkins Road, Laurel, MD 20723, USA.

N. Krupp and E. Roussos, Max-Planck-Institut für Sonnensystemforschung, Max-Planck-Strasse 2, D-37191 Katlenburg-Lindau, Germany. 